\title{
GC-MS analysis of Tasmannia lanceolata Extracts which Inhibit the Growth of the Pathogenic Bacterium Clostridium perfringens
}

\author{
Mitchell Henry Wright ${ }^{1,2}$, Cameron Jay Lee ${ }^{2}$, Megan Sarah Jean Arnold ${ }^{3}$, Joseph Shalom ${ }^{2,4}$, Alan White ${ }^{2}$, \\ Anthony Carlson Greene ${ }^{2}$, lan Edwin Cock ${ }^{2,4}$
}

\section{Mitchell Henry Wright, ${ }^{1,2}$ Cameron Jay Lee, ${ }^{2}$ Megan Sarah Jean Arnold, ${ }^{3}$ Joseph Shalom, 2,4 Alan White, ${ }^{2}$ Anthony Carlson Greene, ${ }^{2}$ Ian Edwin Cock ${ }^{2,4}$}

'Division of Environmental and Biomolecular Systems, Institute of Environmental Health, Oregon Health \& Science University, Portland, Oregon, USA.

${ }^{2} S$ chool of Natural Sciences, Griffith University, Nathan Campus, Queensland, AUSTRALIA.

${ }^{3}$ Eskitis Institute for Drug Discovery, Griffith University, Nathan Campus, Queensland, AUSTRALIA. ${ }^{4}$ Environmental Futures Research Institute, Nathan Campus, Griffith University, Nathan, Queensland 4111, AUSTRALIA.

\section{Correspondence}

Dr. Ian Edwin Cock, School of Natural Sciences, Griffith University,

170 Kessels Rd, Nathan Campus, Queensland, AUSTRALIA

Tel.: +61737357637

E-mail: i.cock@griffith.edu.au

History

- Submission Date: 21-03-2017;

- Review completed: 04-05-2017.

- Accepted Date: 30-05-2017

DOI : 10.5530/pj.2017.5.100

Article Available online

http://www.phcogj.com/v9/i5

Copyright

(C) 2017 Phcog.Net. This is an openaccess article distributed under the terms of the Creative Commons Attribution 4.0 International license.

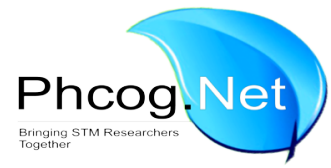

\section{ABSTRACT}

Introduction: Clostridium perfringens is the etiological agent of clostridial myonecrosis and enteritis necroticans. Infections result in exotoxin production, tissue necrosis and unless promptly treated, often result in death. Methods: Tasmannia lanceolata extracts were investigated for $C$. perfringens growth inhibitory activity by disc diffusion analysis and MIC determination. Toxicity was evaluated by Artemia nauplii bioassay and the most potent extracts were phytochemically evaluated by GC-MS headspace analysis. Results: All T. lanceolata berry and leaf extracts displayed potent $C$. perfringens growth inhibition. The berry extracts were more potent growth inhibitors than the corresponding leaf extracts, although the leaf extracts were also potent growth inhibitors. The berry aqueous, methanolic and ethyl acetate extracts were particularly potent growth inhibitors, with MIC values of 654,65 and $329 \mu \mathrm{g} / \mathrm{mL}$ respectively. T. lanceolata leaf also displayed good efficacy, with an MIC of 839, 1255 and $625 \mu \mathrm{g} / \mathrm{mL}$ for the aqueous, methanolic and ethyl acetate extracts respectively. All extracts were nontoxic in the Artemia franciscana bioassay, with $L_{50}$ values substantially $>1000 \mu \mathrm{g} / \mathrm{mL}$. Non-biased GC-MS analysis of the aqueous, methanolic and ethyl acetate berry extracts revealed the presence of high relative levels of a diversity of terpenoids. Conclusions: The lack of toxicity of the $T$. lanceolata extracts and their potent growth inhibitory bioactivity against $C$. perfringens indicates their potential as medicinal agents in the treatment and prevention of clostridial myonecrosis and enteritis necroticans. GC-MS metabolomic profiling studies indicate that these extracts contained a diversity of terpenoids, with monoterpenoids being particularly abundant.

Key words: Winteraceae, Tasmannia lanceolata, Myonecrosis, Enteritis necroticans, Gas gangrene.

\section{INTRODUCTION}

Clostridium perfringens is an endospore-forming Gram-positive, obligate anaerobic bacterium that is found in a variety of habitats, including soils, sewage or as a naturally occurring inhabitant of the intestinal microflora of humans. ${ }^{1}$ It is classed as an opportunistic pathogen and is an etiological agent of clostridial gastroenteritis as well as several other diseases including myonecrosis. $^{2}$ The species $C$. perfringens is divided into five type strains (A-E), each capable of producing exotoxins that are linked to different illnesses of varying severity. These can range from mild food-poisoning to the potentially fatal clostridial myonecrosis (gas gangrene). ${ }^{3}$ Clostridial myonecrosis is a rapidly progressive infection of the soft tissue. The disease is characterised by the necrosis of local muscle and surrounding tissue and can lead to shock and ultimately death, even when promptly treated. ${ }^{1}$ Although several Clostridium spp. can cause gas gangrene, $C$. perfringens is the primary bacterium responsible for the disease (along with C. bifermentans, C. fallax, C. histolyticum, C. novyi,
C. septicum and C. tertium), accounting for up to $80 \%$ of all recorded cases. ${ }^{4}$ The primary methods of treatment are through prompt antibiotic therapy and surgery, however combining these with hyperbaric oxygen can slow the spread of gas gangrene in patients. The strictly anaerobic nature of $C$. perfringens means that when exposed to oxygen, cellular replication and exotoxin production can be inhibited. However, this method of treatment is bacteriostatic and thus must be combined with antibiotic chemotherapy. ${ }^{5}$ Traditional antibiotic therapies offer an effective means of treatment; however, the probing of natural compounds offers an innovative means of treatment and lowers the inherent risk of antibiotic resistance of $C$. perfringens.

Tasmannia lanceolata (Poir.) A.C.Sm (commonly referred to as Tasmanian pepper or mountain pepper berry) is a shrub endemic to the woodlands and cool temperate rainforests of Tasmania and the south-eastern region of the Australian mainland. ${ }^{6}$ It

Cite this article : Wright MH, Lee CJ, Arnold MSJ, Shalom J, White A, Greene AC, Cock IE. GC-MS analysis of Tasmannia lanceolata extracts which inhibit the growth of the pathogenic bacterium Clostridium perfringens. Pharmacog J. 2017;9(5):626-37. 
is a medium to large shrub that varies between $2-5 \mathrm{~m}$ in height. Individual plants are unisexual, having either male or female flowers. The stems, branches and twigs are red in colour. The aromatic leaves are lanceolate to narrowly elliptical in shape (4-12 cm long, $0.7-2 \mathrm{~cm}$ wide) with a distinctly pale under surface. Small creamy-white unisexual flowers appear during the summer months. These develop into small fleshy black 2 lobed berries (5-8 mm wide) during autumn.

The berries, leaves and bark of this species have historical uses as a food and as a medicinal plant. ${ }^{6,7}$ When the berry is air dried, it forms a small, hard peppercorn which is suitable for milling or crushing. The berry has a pleasant spicy flavour and sharp aroma. T. lanceolata was used as flavouring agent by Australian Aborigines and more recently by European settlers. Historically, the leaves have been used as an herb and the berries have been used as a spice. Australian Aborigines also used T. lanceolata as a therapeutic agent to treat stomach disorders and as an emetic, as well as general usage as a tonic. ${ }^{7}$ T. lanceolata was also used traditionally for the treatment and cure of skin disorders, venereal diseases, colic, stomach aches and as a quinine substitute. ${ }^{7}$ Later, European colonists also recognized the therapeutic potential of T. lanceolata and the bark was used as a common substitute for other herbal remedies (including those derived from the related South American Winteraceae species, Drimys wintera (winter bark) ${ }^{7}$ to treat scurvy due to its high anti-antioxidant content. ${ }^{7,8}$

Despite its ethnobotanical usage, there have been limited rigorous scientific studies into the therapeutic properties of T. lanceolata. Recent studies have demonstrated the high antioxidant content of $T$. lanceolata fruit and leaves. ${ }^{8}$ It has been postulated that this high anti-oxidant content may provide therapeutic effects for this plant. ${ }^{7}$ Indeed, studies within our laboratory have reported potent inhibition of bacterial growth by T. lanceolata berries, leaves and peppercorns against panels of pathogenic and food spoilage bacteria. ${ }^{9}$ T. lanceolata extracts can also inhibit the growth of a bacterial trigger of rheumatoid arthritis (P. mirabilis) ${ }^{10}$

However, despite the documented ability of T. lanceolata to inhibit the growth of many bacterial species, to our knowledge there have been no similar studies against Clostridium perfringens. The current study was undertaken to test T. lanceolata berry and leaf extracts for the ability to inhibit the growth of this pathogen.

\section{MATERIALS AND METHODS}

\section{T. lanceolata samples and extraction}

Dried Tasmannia lanceolata (Poir.) A.C.Sm berry (without seed) and leaf materials were obtained from Go Wild Harvest, Australia. The material was thoroughly dried using a Sunbeam food dehydrator and stored at $-30^{\circ} \mathrm{C}$ until use. The dried plant materials were thawed and freshly ground to a coarse powder prior to extraction. Individual $1 \mathrm{~g}$ quantities were extracted by weighing each powdered plant part into each of 5 tubes and adding $50 \mathrm{~mL}$ of methanol, water, ethyl acetate, chloroform or hexane respectively. All solvents were obtained from Ajax, Australia and were AR grade. The berry and leaf material was extracted in each solvent for $24 \mathrm{~h}$ at $4^{\circ} \mathrm{C}$ with gentle shaking. The extracts were filtered through filter paper (Whatman No. 54) under vacuum followed by drying by rotary evaporation in an Eppendorf concentrator 5301. The resultant dry extracts were weighed and redissolved in $10 \mathrm{~mL}$ deionised water (containing 1\% DMSO).

\section{Qualitative phytochemical studies}

Phytochemical analysis of the extracts for the presence of alkaloids, anthraquinones, cardiac glycosides, flavonoids, phenolic compounds, phytosterols, saponins tannins and triterpenoids were conducted by previously described assays. ${ }^{11,12}$

\section{Antibacterial screening}

\section{Clinical Clostridium perfringens screening}

A clinical strain of Clostridium perfringens was supplied by Ms. Jane Gifkins (Griffith University) and was originally isolated and verified by John Bates (Queensland Department of Health). Cultures were grown and maintained using a thioglycolate liquid media (Oxoid Ltd., Australia). All growth studies were performed using nutrient agar (Oxoid Ltd., Australia) under induced anaerobic conditions using anaerobic jars and AnaeroGen $^{\mathrm{na}} 3.5 \mathrm{~L}$ atmospheric generation systems (Thermo Scientific) as previously described. ${ }^{[13]}$ Incubation was at $30^{\circ} \mathrm{C}$ and the stock culture was subcultured and maintained in thioglycolate liquid media at $4{ }^{\circ} \mathrm{C}$.

\section{Evaluation of antimicrobial activity}

Antimicrobial activity of all plant extracts was determined using a modified disc diffusion assay. ${ }^{14,15}$ Briefly, $100 \mu \mathrm{L}$ of the test bacteria were grown in $10 \mathrm{~mL}$ of fresh nutrient broth media until they reached a count of $\sim 10^{8}$ cells $/ \mathrm{mL}$. A volume of $100 \mu \mathrm{L}$ of bacterial suspension was spread onto nutrient agar plates and extracts were tested for antibacterial activity using $5 \mathrm{~mm}$ sterilised filter paper discs. Discs were infused with $10 \mu \mathrm{L}$ of the test sample, allowed to dry and placed onto inoculated plates. The plates were allowed to stand at $4{ }^{\circ} \mathrm{C}$ for $2 \mathrm{~h}$ before incubation at $30^{\circ} \mathrm{C}$ for $24 \mathrm{~h}$. The diameters of the inhibition zones were measured in millimetres. All measurements were to the closest whole millimetre. Each assay was performed in at least triplicate. Mean values $( \pm$ SEM) are reported in this study. Standard discs of penicillin $(2 \mu \mathrm{g})$ and ampicillin $(10 \mu \mathrm{g})$ were obtained from Oxoid Ltd., Australia and used as positive controls for antibacterial activity. Filter discs infused with $10 \mu \mathrm{L}$ of distilled water were used as a negative control.

\section{Minimum inhibitory concentration (MIC) determination}

The minimum inhibitory concentrations (MIC) of the extracts was determined as previously described ${ }^{16,}{ }^{17}$ Briefly, the plant extracts were diluted in deionised water and tested across a range of concentrations. Discs were infused with $10 \mu \mathrm{L}$ of the extract dilutions, allowed to dry and placed onto inoculated plates. The assay was performed as outlined above and graphs of the zone of inhibition versus concentration were plotted. Linear regression was used to calculate the MIC values.

\section{Toxicity screening \\ Reference toxin for toxicity screening}

Potassium dichromate $\left(\mathrm{K}_{2} \mathrm{Cr}_{2} \mathrm{O}_{7}\right)$ (AR grade, Chem-Supply, Australia) was prepared as a $4 \mathrm{mg} / \mathrm{mL}$ solution in distilled water and serially diluted in artificial seawater for use in the Artemia franciscana nauplii bioassay.

\section{Artemia franciscana nauplii toxicity screening}

Toxicity was tested using a modified Artemia franciscana nauplii lethality assay. ${ }^{18,19}$ Briefly, $400 \mu \mathrm{L}$ of seawater containing approximately 43 (mean $43.2, \mathrm{n}=155, \mathrm{SD} 14.5)$ A. franciscana nauplii were added to wells of a 48 well plate and immediately used for bioassay. A volume of $400 \mu \mathrm{L}$ of diluted plant extracts or the reference toxin were transferred to the wells and incubated at $25 \pm 1{ }^{\circ} \mathrm{C}$ under artificial light (1000 Lux). A negative control ( $400 \mu \mathrm{L}$ seawater) was run in triplicate for each plate. All treatments were performed in at least triplicate. The wells were checked at regular intervals and the number of dead counted. The nauplii were considered dead if no movement of the appendages was observed within 10 seconds. After $24 \mathrm{~h}$ all nauplii were sacrificed and counted to determine the total $\%$ mortality per well. The $\mathrm{LC}_{50}$ with $95 \%$ confidence limits was calculated using probit analysis. 


\section{Non-targeted GC-MS head space analysis}

Separation and quantification of phytochemical components were performed using a Shimadzu GC-2010 plus (USA) linked to a Shimadzu MS TQ8040 (USA) mass selective detector system as previously described. ${ }^{20}$ Briefly, the system was equipped with a Shimadzu auto-sampler AOC5000 plus (USA) fitted with a solid phase micro-extraction fibre (SPME) handling system utilising a Supelco (USA) divinyl benzene/carbowax/ polydimethylsiloxane (DVB/CAR/PDMS). Chromatographic separation was accomplished using a 5\% phenyl, 95\% dimethylpolysiloxane (30 m x $0.25 \mathrm{~mm}$ id x $0.25 \mathrm{um}$ ) capillary column (Restek USA). Helium (99.999\%) was employed as a carrier gas at a flow rate of $0.79 \mathrm{~mL} / \mathrm{min}$. The injector temperature was set at $230^{\circ} \mathrm{C}$. Sampling utilised a SPME cycle which consisted of an agitation phase at $500 \mathrm{rpm}$ for a period of $5 \mathrm{sec}$. The fibre was exposed to the sample for $10 \mathrm{~min}$ to allow for absorption and then desorbed in the injection port for $1 \mathrm{~min}$ at $250{ }^{\circ} \mathrm{C}$. The initial column temperature was held at $30^{\circ} \mathrm{C}$ for $2 \mathrm{~min}$, increased to $140{ }^{\circ} \mathrm{C}$ for $5 \mathrm{~min}$, then increased to $270{ }^{\circ} \mathrm{C}$ over a period of $3 \mathrm{mins}$ and held at that temperature for the duration of the analysis. The GC-MS interface was maintained at $200{ }^{\circ} \mathrm{C}$ with no signal acquired for a min after injection in split-less mode. The mass spectrometer was operated in the electron ionisation mode at $70 \mathrm{eV}$. The analytes were then recorded in total ion count (TIC) mode. The TIC was acquired after a min and for duration of 45 mins utilising a mass range of $45-450 \mathrm{~m} / \mathrm{z}$.

\section{Statistical analysis}

Data is expressed as the mean \pm SEM of at least three independent experiments.

\section{RESULTS}

Extraction of $1 \mathrm{~g}$ of the $T$. lanceolata plant materials with various solvents yielded dried plant extracts ranging from $17 \mathrm{mg}$ (T. lanceolata leaf ethyl acetate extract) to $171 \mathrm{mg}$ (methanolic T. lanceolata fruit extract) (Table 1). Aqueous and methanolic extracts generally gave higher yields of dried extracted material compared to ethyl acetate extracts. The dried extracts were resuspended in $10 \mathrm{~mL}$ of deionised water (containing $1 \%$ DMSO) resulting in the extract concentrations shown in Table 1.

Qualitative phytochemical studies showed little difference between the aqueous and methanolic extracts, however there were notable differences between these extracts and the ethyl acetate extracts. High levels of phenolics (both water soluble and insoluble) were extracted in the aqueous and methanolic samples. There were substantially lower levels detected in the corresponding ethyl acetate extracts. Similarly, there was a lower level of flavonoids detected in the ethyl acetate extracts than the corresponding aqueous and methanolic extracts. Triterpenes were detected in both methanolic and ethyl acetate extracts although they were absent in the aqueous extracts.

To measure the inhibitory activity of the crude plant extracts against C. perfringens, $10 \mu \mathrm{L}$ aliquots of each extract were screened using a disc diffusion assay. The bacterial growth was inhibited by all of the 6 extracts tested (Figure 1). The methanolic berry extract was the most potent inhibitor of growth, with inhibition zones of $16.3 \pm 0.3 \mathrm{~mm}$. This compares favourably with the penicillin and ampicillin controls, which had inhibitory zones of $12.3 \pm 0.3 \mathrm{~mm}$ and $13.0 \pm 1.0 \mathrm{~mm}$ respectively. The aqueous and methanolic extracts showed greater zones of inhibition than the ethyl acetate extracts, with inhibitory zones $\geq 11 \mathrm{~mm}$.

The antimicrobial efficacy was further quantified by determining the MIC values (Table 2). All the extracts were determined to be potent inhibitors

Table 1: The mass of dried extracted material, the concentration after resuspension in deionised water and qualitative phytochemical screenings of the $T$. lanceolata extracts.

\begin{tabular}{|c|c|c|c|c|c|c|c|c|c|c|c|c|c|c|c|}
\hline 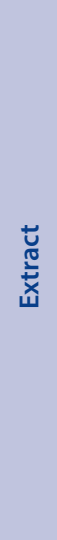 & 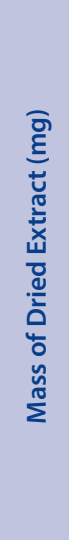 & 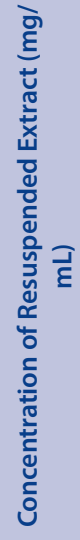 & $\begin{array}{l}\frac{\tilde{U}}{\overline{0}} \\
\frac{c}{\Phi} \\
\frac{\tilde{c}}{0} \\
\frac{\pi}{\tilde{0}} \\
\stackrel{0}{0}\end{array}$ & 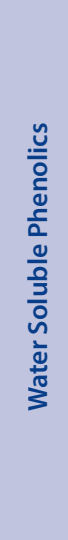 & 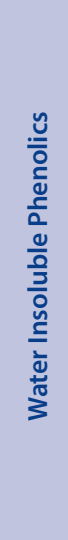 & 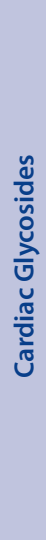 & 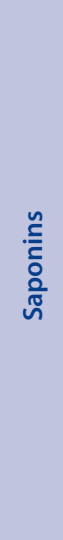 & 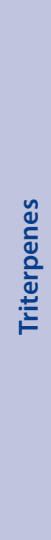 & 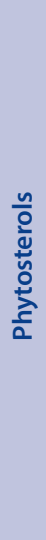 & 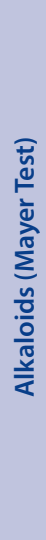 & 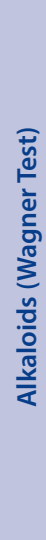 & $\begin{array}{l}\text { 음 } \\
\frac{0}{0} \\
\frac{\pi}{0} \\
\end{array}$ & 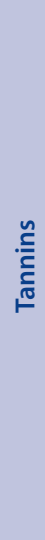 & 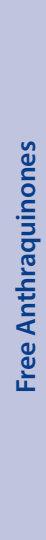 & 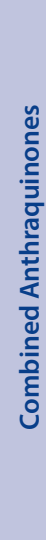 \\
\hline BW & 111 & 11.1 & +++ & +++ & +++ & - & - & - & - & - & - & +++ & - & - & - \\
\hline $\mathrm{BM}$ & 171 & 17.1 & +++ & +++ & +++ & - & ++ & + & - & - & - & +++ & - & - & - \\
\hline $\mathrm{BE}$ & 56.7 & 5.7 & + & + & ++ & - & + & ++ & - & - & - & ++ & - & - & - \\
\hline LW & 134 & 13.4 & +++ & +++ & +++ & - & ++ & - & - & - & - & +++ & - & - & - \\
\hline LM & 144 & 14.4 & +++ & +++ & +++ & - & +++ & + & - & - & - & +++ & - & - & - \\
\hline LE & 17 & 1.7 & + & + & ++ & - & - & + & - & - & - & ++ & - & - & - \\
\hline
\end{tabular}

+++ indicates a large response; ++ indicates a moderate response; + indicates a minor response; - indicates no response in the assay. BW $=$ aqueous $T$. lanceolata berry extract; $\mathrm{BM}=$ methanolic $T$. lanceolata berry extract; $\mathrm{BE}=$ ethyl acetate T. lanceolata berry extract; $\mathrm{LW}=$ aqueous $T$. lanceolata leaf extract; LM = methanolic T. lanceolata leaf extract; LE = ethyl acetate T. lanceolata leaf extract. 


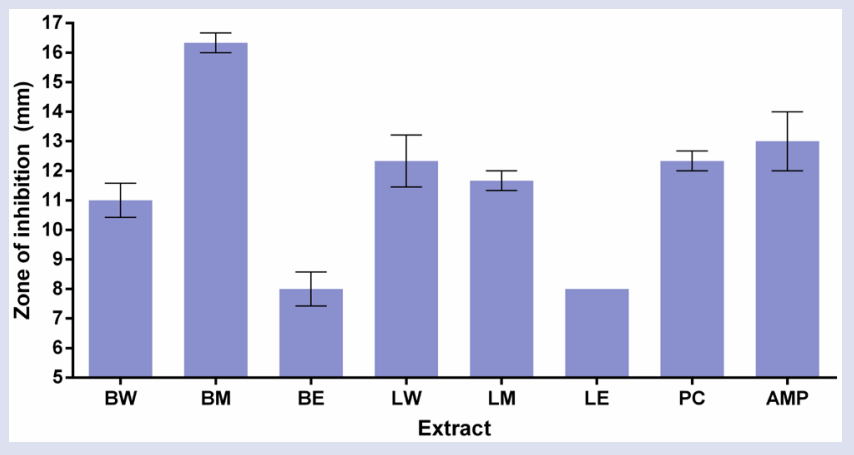

Figure 1: Growth inhibitory activity of T. lanceolata extracts against the C. perfringens clinical isolate measured as zones of inhibition ( $\mathrm{mm}$ ). BW $=$ aqueous $T$. lanceolata berry extract; $\mathrm{BM}=$ methanolic $T$. lanceolata berry extract; $\mathrm{BE}=$ ethyl acetate $T$. lanceolata berry extract; $\mathrm{LW}=$ aqueous $T$. lanceolata leaf extract; LM = methanolic $T$. lanceolata leaf extract; $\mathrm{LE}=$ ethyl acetate $T$. lanceolata leaf extract; $\mathrm{PC}=$ penicillin $(2 \mu \mathrm{g})$; AMP $=$ ampicillin $(10 \mu \mathrm{g})$. Results are expressed as mean zones of inhibition \pm SEM.
Table 2: Minimum inhibitory concentration $(\mu \mathrm{g} / \mathrm{mL})$ of the plant extracts and $\mathrm{LC}_{50}$ values $(\mu \mathrm{g} / \mathrm{mL})$ in the Artemia nauplii bioassay.

\begin{tabular}{ccc} 
Extract & MIC & LC $_{50}$ \\
BW & 654 & 2.376 \\
BM & 65 & 3.573 \\
BE & 329 & 3.132 \\
LW & 839 & 2.665 \\
LM & 1.255 & 3.096 \\
LE & 625 & 1.766 \\
PC & ND & 186 \\
SW & ND & - \\
\hline
\end{tabular}

Numbers indicate the mean MIC and $\mathrm{LC}_{50}$ values of triplicate determinations. - indicates no inhibition. $\mathrm{BW}=$ aqueous $T$. lanceolata berry extract; $\mathrm{BM}=$ methanolic T. lanceolata berry extract; $\mathrm{BE}=$ ethyl acetate $T$. lanceolata berry extract; $\mathrm{LW}=$ aqueous $T$. lanceolata leaf extract; $\mathrm{LM}=$ methanolic $T$. lanceolata leaf extract; $\mathrm{LE}=$ ethyl acetate $T$. lanceolata leaf extract; $\mathrm{PC}=$ potassium dichromate control; $\mathrm{SW}=$ artificial seawater negative control; $\mathrm{ND}=$ the indicated test was not performed.

of C. perfringens growth, with MIC $<1000 \mu \mathrm{g} / \mathrm{mL}$ for all extracts except the aqueous leaf extract. Even that extract had a relatively low MIC $(1255 \mu \mathrm{g} / \mathrm{mL})$, indicating antibacterial efficacy. The T. lanceolata berry extracts were more potent inhibitors than were the corresponding leaf extracts. Indeed, a MIC of $65 \mu \mathrm{g} / \mathrm{mL}$ was determined for the methanolic T. lanceolata berry extract. This is particularly noteworthy as it equates to a mass of less than $0.7 \mu \mathrm{g}$ infused into the disc (compared with 2 and $10 \mu \mathrm{g}$ for the penicillin and ampicillin controls respectively).

All extracts were initially screened at $2000 \mu \mathrm{g} / \mathrm{mL}$ in the assay (Figure 2). For comparison, the reference toxin potassium dichromate $(1000 \mu \mathrm{g} / \mathrm{mL})$ was also tested in the bioassay. The potassium dichromate reference toxin was rapid in its onset, promoting nauplii death within the first $3 \mathrm{~h}$ of exposure with $100 \%$ mortality evident following 4-5 h (unpublished results). Similarly, all the $T$. lanceolata extracts displayed significant mortality rates following $24 \mathrm{~h}$ exposure (>50\%).

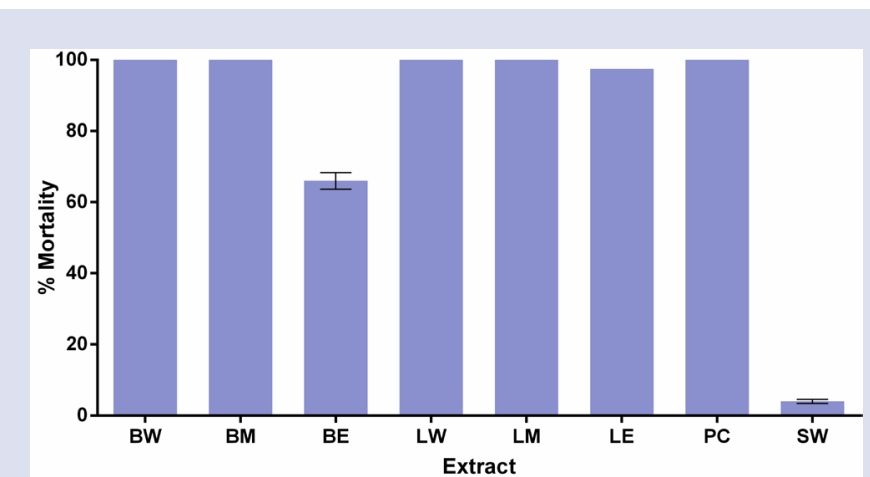

Figure 2: The lethality of the T. lanceolata extracts $(2000 \mu \mathrm{g} / \mathrm{mL})$ and the potassium dichromate control $(1000 \mu \mathrm{g} / \mathrm{mL})$ towards Artemia franciscana nauplii after $24 \mathrm{~h}$ exposure. BW = aqueous $T$. lanceolata berry extract; $\mathrm{BM}=$ methanolic $T$. lanceolata berry extract; $\mathrm{BE}=$ ethyl acetate $T$. lanceolata berry extract; $\mathrm{LW}=$ aqueous $T$. lanceolata leaf extract; $\mathrm{LM}=$ methanolic $T$. lanceolata leaf extract; $\mathrm{LE}=$ ethyl acetate $T$. lanceolata leaf extract; $P C=$ potassium dichromate control; $S W=$ artificial seawater control. Results are expressed as mean $\%$ mortality \pm SEM.

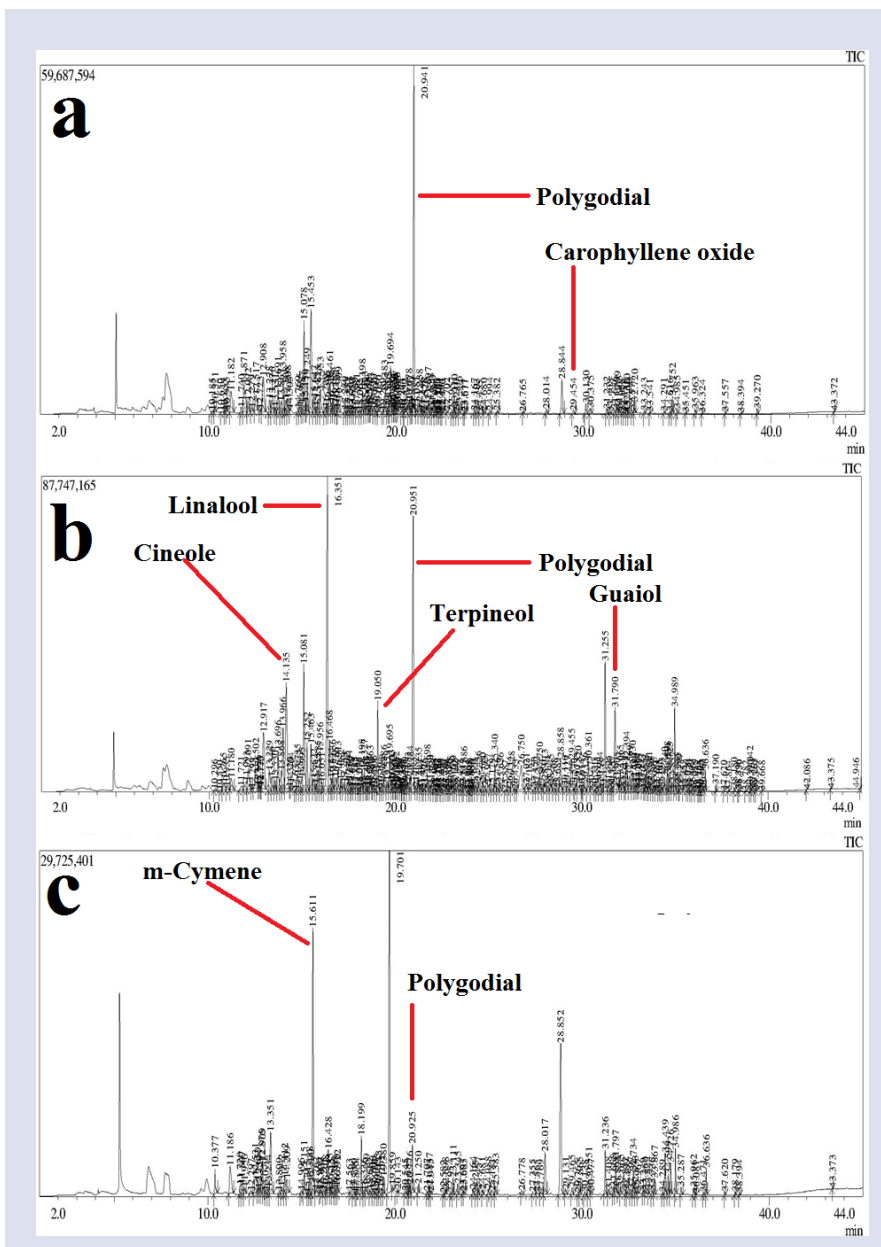

Figure 3: Head space gas chromatograms of $0.5 \mu \mathrm{L}$ injections of T. lanceolata berry (a) aqueous, (b) methanolic and (c) ethyl acetate extracts. The extracts were dried and resuspended in methanol. Chromatography conditions were as described in the methods section. 
Table 3: Qualitative GC-MS headspace analysis of the $T$. lanceolata berry extracts, elucidation of empirical formulas and putative identification of each compound.

\begin{tabular}{|c|c|c|c|c|c|c|}
\hline Molecular Mass & Molecular Formula & R.Time & Name & M & w & $\mathrm{E}$ \\
\hline 110 & $\mathrm{C}_{6} \mathrm{H}_{6} \mathrm{O}_{2}$ & 10.19 & Acetylfuran & & + & \\
\hline 151 & $\mathrm{C}_{8} \mathrm{H}_{9} \mathrm{NO}_{2}$ & 10.375 & Methyl N-hydroxybenzenecarboximidoate & & & + \\
\hline 128 & $\mathrm{C}_{8} \mathrm{H}_{6}{ }_{6} \mathrm{O}$ & 10.55 & 2,4,4-trimethyl-cyclopentanol & + & & \\
\hline 130 & $\mathrm{C}_{7} \mathrm{H}_{14} \mathrm{O}_{2}$ & 10.765 & Methyl caproate & + & & \\
\hline 130 & $\mathrm{C}_{8} \mathrm{H}_{18} \mathrm{O}$ & 10.892 & 4-Methyl-4-heptanol & & + & \\
\hline 170 & $\mathrm{C}_{12} \mathrm{H}_{26}$ & 10.935 & 3,8-Dimethyldecane & + & + & \\
\hline 128 & $\mathrm{C}_{8} \mathrm{H}_{16} \mathrm{O}$ & 11.18 & 4-Methyl-2-heptanone & + & + & + \\
\hline 126 & $\mathrm{C}_{9} \mathrm{H}_{18}$ & 11.721 & 1,1,3,4-Tetramethylcyclopentane & + & + & + \\
\hline 156 & $\mathrm{C}_{10} \mathrm{H}_{20} \mathrm{O}$ & 11.805 & 2,2,5,5-Tetramethyl-3-hexanone & & & + \\
\hline 110 & $\mathrm{C}_{6} \mathrm{H}_{6} \mathrm{O}_{2}$ & 11.87 & 2-Formyl-5-methylfuran & & + & \\
\hline 120 & $\mathrm{C}_{9} \mathrm{H}_{12}$ & 11.993 & p-Ethylmethylbenzene & + & & \\
\hline 120 & $\mathrm{C}_{9} \mathrm{H}_{12}$ & 12.12 & m-Ethyltoluene & & & \\
\hline 156 & $\mathrm{C}_{10} \mathrm{H}_{20} \mathrm{O}$ & 12.299 & Decanal & + & & + \\
\hline 154 & $\mathrm{C}_{10} \mathrm{H}_{18} \mathrm{O}$ & 12.28 & 2,2,6-Trimethyl-6-vinyltetrahydropyran & & + & \\
\hline 114 & $\mathrm{C}_{8} \mathrm{H}_{18}$ & 12.502 & 2,4-Dimethylhexane & + & + & \\
\hline 186 & $\mathrm{C}_{9} \mathrm{H}_{14} \mathrm{O}_{4}$ & 12.535 & alpha.-D-Xylo-Hex-5-enofuranose, 5,6-dideoxy-1,2-O-(1-methylethylidene)- & & & + \\
\hline 284 & $\mathrm{C}_{16} \mathrm{H}_{28} \mathrm{O}_{4}$ & 12.626 & Succinic acid, 2-methylpent-3-yl trans-he & + & & + \\
\hline 126 & $\mathrm{C}_{8} \mathrm{H}_{14} \mathrm{O}$ & 12.72 & 6-Methyl-5-hepten-2-one & + & + & + \\
\hline 146 & $\mathrm{C}_{7} \mathrm{H}_{14} \mathrm{O}_{3}$ & 12.773 & Methyl 2-hydroxy-4-methylpentanoate & + & & \\
\hline 170 & $\mathrm{C}_{12} \mathrm{H}_{26}$ & 13.229 & Dodecane & + & + & + \\
\hline 128 & $\mathrm{C}_{8} \mathrm{H}_{16} \mathrm{O}$ & 13.235 & Octanal & & & \\
\hline 142 & $\mathrm{C}_{10} \mathrm{H}_{22}$ & 13.53 & 3,3,5-Trimethylheptane & + & + & \\
\hline 170 & $\mathrm{C}_{12} \mathrm{H}_{26}$ & 13.696 & 3,4,5,6-Tetramethyloctane & + & + & \\
\hline 120 & $\mathrm{C}_{9} \mathrm{H}_{12}$ & 13.868 & Cumene & + & + & + \\
\hline 140 & $\mathrm{C}_{9} \mathrm{H}_{16} \mathrm{O}$ & 13.94 & Ethanone, 1-(2,2-dimethylcyclopentyl)- & & & + \\
\hline 142 & $\mathrm{C}_{10} \mathrm{H}_{22}$ & 13.966 & 3,3-Dimethyloctane & + & + & \\
\hline 154 & $\mathrm{C}_{10} \mathrm{H}_{18} \mathrm{O}$ & 14.135 & Cineole & + & + & \\
\hline 140 & $\mathrm{C}_{9} \mathrm{H}_{16} \mathrm{O}$ & 14.21 & 1,1,3-Trimethyl-2-cyclohexanone & & + & + \\
\hline 136 & $\mathrm{C}_{10} \mathrm{H}_{16}$ & 14.404 & alpha.-Pinene & + & & \\
\hline 136 & $\mathrm{C}_{10} \mathrm{H}_{16}$ & 14.735 & beta.-Ocimene & + & & \\
\hline 244 & $\mathrm{C}_{13} \mathrm{H}_{24} \mathrm{O}_{4}$ & 14.847 & Oxalic acid, isohexyl neopentyl ester & + & + & \\
\hline 278 & $\mathrm{C}_{14} \mathrm{H}_{30} \mathrm{O}_{3} \mathrm{~S}$ & 14.995 & Sulfurous acid, nonyl pentyl ester & + & + & \\
\hline 114 & $\mathrm{C}_{8} \mathrm{H}_{18}$ & 15.081 & 3,3-Dimethylhexane & + & + & \\
\hline 120 & $\mathrm{C}_{8} \mathrm{H}_{8} \mathrm{O}$ & 15.15 & Bicyclo[4.2.0]octa-1,3,5-trien-7-ol & & & + \\
\hline 242 & $\mathrm{C}_{13} \mathrm{H}_{22} \mathrm{O}_{4}$ & 15.463 & Ethyl 2-(5-methyl-5-vinyltetrahydrofuran-2-yl)propan-2-yl carbonate & + & + & \\
\hline 212 & $\mathrm{C}_{14} \mathrm{H}_{12} \mathrm{O}_{2}$ & 15.615 & m-Toluic acid, phenyl ester & & + & + \\
\hline 134 & $\mathrm{C}_{10} \mathrm{H}_{14}$ & 15.622 & m-Cymene & + & & \\
\hline 200 & $\mathrm{C}_{13} \mathrm{H}_{28} \mathrm{O}$ & 15.744 & 11-Methyldodecanol & + & + & \\
\hline 134 & $\mathrm{C}_{10} \mathrm{H}_{14}$ & 15.835 & 1,2-Dimethyl-4-ethylbenzene & + & + & + \\
\hline 170 & $\mathrm{C}_{10} \mathrm{H}_{18} \mathrm{O}_{2}$ & 15.956 & Linalool oxide & + & + & + \\
\hline 172 & $\mathrm{C}_{10} \mathrm{H}_{20} \mathrm{O}_{2}$ & 16.051 & 3-(Hydroxymethyl)-2-nonanone & + & & \\
\hline 228 & $\mathrm{C}_{13} \mathrm{H}_{24} \mathrm{O}_{3}$ & 16.23 & Carbonic acid, nonyl prop-1-en-2-yl ester & & + & \\
\hline
\end{tabular}

Continued... 
Table 3: Cont'd.

\begin{tabular}{|c|c|c|c|c|c|c|}
\hline Molecular Mass & Molecular Formula & R.Time & Name & M & w & $\mathrm{E}$ \\
\hline 154 & $\mathrm{C}_{10} \mathrm{H}_{18} \mathrm{O}$ & 16.351 & Linalool & + & + & + \\
\hline 170 & $\mathrm{C}_{12} \mathrm{H}_{26}$ & 16.468 & 4-Methylundecane & + & + & + \\
\hline 140 & $\mathrm{C}_{10} \mathrm{H}_{20}$ & 16.544 & 1-Ethyl-1,4-dimethylcyclohexane & + & + & \\
\hline 156 & $\mathrm{C}_{11} \mathrm{H}_{24}$ & 16.636 & 6-Ethyl-2-methyloctane & + & & \\
\hline 134 & $\mathrm{C}_{10} \mathrm{H}_{14}$ & 16.786 & 3,7,7-Trimethyl-1,3,5-cycloheptatriene & + & + & \\
\hline 134 & $\mathrm{C}_{10} \mathrm{H}_{14}$ & 16.913 & p-Cymene & + & + & + \\
\hline 156 & $\mathrm{C}_{10} \mathrm{H}_{20} \mathrm{O}$ & 17.233 & 1-Methyl-4-(1-methylethyl)cyclohexanol & + & + & \\
\hline 156 & $\mathrm{C}_{10} \mathrm{H}_{20} \mathrm{O}$ & 17.402 & Neoisocarquejanol & + & & \\
\hline 152 & $\mathrm{C}_{10} \mathrm{H}_{16} \mathrm{O}$ & 17.491 & 4(10)-Thujen-3-ol & + & + & \\
\hline 184 & $\mathrm{C}_{11} \mathrm{H}_{20} \mathrm{O}_{2}$ & 17.56 & 2,2,6,6-Tetramethyl-3,5-heptanedione & + & + & + \\
\hline 166 & $\mathrm{C}_{12} \mathrm{H}_{22}$ & 18.036 & 1-Hexyl-1-cyclohexene & + & & \\
\hline 154 & $\mathrm{C}_{10} \mathrm{H}_{18} \mathrm{O}$ & 18.327 & cis-2-Norbornanol & + & & + \\
\hline 144 & $\mathrm{C}_{8} \mathrm{H}_{16} \mathrm{O}_{2}$ & 18.47 & Octanoic acid & & & + \\
\hline 170 & $\mathrm{C}_{10} \mathrm{H}_{10} \mathrm{O}_{2}$ & 18.565 & trans-Linalool 3,7-oxide & + & & \\
\hline 168 & $\mathrm{C}_{12} \mathrm{H}_{24}$ & 18.663 & 4,6,8-Trimethyl-1-nonene & + & + & \\
\hline 130 & $\mathrm{C}_{8} \mathrm{H}_{18} \mathrm{O}$ & 18.913 & 2,5-Dimethyl-2-hexanol & + & + & \\
\hline 154 & $\mathrm{C}_{10} \mathrm{H}_{18} \mathrm{O}$ & 19.05 & .alpha.-Terpineol & + & + & + \\
\hline 152 & $\mathrm{C}_{10} \mathrm{H}_{16} \mathrm{O}$ & 19.283 & Myrtenol & + & + & \\
\hline 156 & $\mathrm{C}_{10} \mathrm{H}_{20} \mathrm{O}$ & 19.559 & 5-Methyl-3-propyl-2-hexanone & + & + & \\
\hline 134 & $\mathrm{C}_{9} \mathrm{H}_{10} \mathrm{O}$ & 19.695 & 2-methyl-2-phenyl-oxirane & + & + & + \\
\hline 156 & $\mathrm{C}_{11} \mathrm{H}_{24}$ & 19.803 & 2,3,7-Trimethyloctane & + & + & \\
\hline 184 & $\mathrm{C}_{13} \mathrm{H}_{28}$ & 20.032 & 4,8-dimethyl-undecane & + & + & \\
\hline 196 & $\mathrm{C}_{12} \mathrm{H}_{20} \mathrm{O}_{2}$ & 20.155 & Nerol acetate & + & & \\
\hline 184 & $\mathrm{C}_{13} \mathrm{H}_{28}$ & 20.445 & 3,3,5-trimethyl-decane & + & + & \\
\hline 252 & $\mathrm{C}_{17} \mathrm{H}_{32} \mathrm{O}$ & 20.675 & 13-Heptadecyn-1-ol & & + & \\
\hline 296 & $\mathrm{C}_{20} \mathrm{H}_{40} \mathrm{O}$ & 20.683 & Phytol & + & & \\
\hline 232 & $\mathrm{C}_{15} \mathrm{H}_{20} \mathrm{O}_{2}$ & 20.784 & 2,2,3,3-Tetramethylcyclopropanecarboxylic acid, 4-methylphenyl ester & + & + & \\
\hline 234 & $\mathrm{C}_{15} \mathrm{H}_{22} \mathrm{O}_{2}$ & 20.951 & polygodial & + & + & + \\
\hline 184 & $\mathrm{C}_{13} \mathrm{H}_{28}$ & 21.082 & 4-methyl-dodecane & + & + & \\
\hline 158 & $\mathrm{C}_{9} \mathrm{H}_{18} \mathrm{O}_{2}$ & 21.235 & Nonanoic acid & + & + & + \\
\hline 114 & $\mathrm{C}_{8} \mathrm{H}_{18}$ & 21.698 & 3,3-Dimethylhexane & + & + & \\
\hline 188 & $\mathrm{C}_{10} \mathrm{H}_{20} \mathrm{O}_{3}$ & 21.83 & Butyl butoxyacetate & + & + & + \\
\hline 198 & $\mathrm{C}_{14} \mathrm{H}_{30}$ & 22.083 & 4,6-Dimethyldodecane & + & + & \\
\hline 150 & $\mathrm{C}_{10} \mathrm{H}_{14} \mathrm{O}$ & 22.165 & (2E,3Z)-2-Ethylidene-6-methyl-3,5-heptadienal & + & + & \\
\hline 222 & $\mathrm{C}_{16} \mathrm{H}_{30}$ & 22.408 & (2-Cyclohexyl-1-methylpropyl)cyclohexane & + & + & \\
\hline 140 & $\mathrm{C}_{9} \mathrm{H}_{16} \mathrm{O}$ & 22.708 & 2,3,4,5-Tetramethylcyclopent-2-en-1-ol & + & + & + \\
\hline 194 & $\mathrm{C}_{12} \mathrm{H}_{18} \mathrm{O}_{2}$ & 22.876 & 2-Pinen-10-ol, acetate & + & & \\
\hline 212 & $\mathrm{C}_{15} \mathrm{H}_{32}$ & 22.955 & 2,6,11-Trimethyldodecane & + & + & + \\
\hline 158 & $\mathrm{C}_{10} \mathrm{H}_{22} \mathrm{O}$ & 23.215 & 2-Propyl-1-heptanol & & + & \\
\hline 168 & $\mathrm{C}_{12} \mathrm{H}_{24}$ & 23.473 & 4-Methyl-1-undecene & + & & \\
\hline 286 & $\mathrm{C}_{16} \mathrm{H}_{30} \mathrm{O}_{4}$ & 23.619 & $\begin{array}{l}\text { 2,2,4-Trimethyl-1,3-pentanediol diisobutyrate } \$ \text { Propanoic acid, 2-methyl-, } \\
\text { 2,2-dimethyl-1-(1-methylethyl)-1,3-propanediyl ester }\end{array}$ & + & + & \\
\hline
\end{tabular}

Continued... 
Table 3: Cont'd.

\begin{tabular}{|c|c|c|c|c|c|c|}
\hline Molecular Mass & Molecular Formula & R.Time & Name & M & W & $\mathrm{E}$ \\
\hline 164 & $\mathrm{C}_{10} \mathrm{H}_{12} \mathrm{O}_{2}$ & 23.686 & p-Eugenol & + & & + \\
\hline 180 & $\mathrm{C}_{10} \mathrm{H}_{12} \mathrm{O}_{3}$ & 24.01 & beta.-Phenyllactic acid methyl ester & + & & \\
\hline 216 & $\mathrm{C}_{12} \mathrm{H}_{24} \mathrm{O}_{3}$ & 24.17 & Propanoic acid, 2-methyl-, 3-hydroxy-2,2,4-trimethylpentyl ester & + & + & + \\
\hline 241 & $\mathrm{C}_{13} \mathrm{H}_{23} \mathrm{NO}_{3}$ & 24.305 & 4-t-Butyl-2-(1-methyl-2-nitroethyl)cyclohexanone & & + & + \\
\hline 204 & $\mathrm{C}_{15} \mathrm{H}_{24}$ & 24.344 & .alpha.-Cubebene & + & & \\
\hline 180 & $\mathrm{C}_{12} \mathrm{H}_{20} \mathrm{O}$ & 24.456 & 4a,5-Dimethyloctahydro-2(1H)-naphthalenone & + & & + \\
\hline 210 & $\mathrm{C}_{14} \mathrm{H}_{26} \mathrm{O}$ & 24.68 & (6Z)-3,7-Dimethyl-6,11-dodecadien-1-ol & + & + & + \\
\hline 204 & $\mathrm{C}_{15} \mathrm{H}_{24}$ & 24.773 & $\begin{array}{l}\text { Cyclohexane, 1-ethenyl-1-methyl-2,4-bis(1-methylethenyl)-, [1S-(1.alpha.,2. } \\
\text { beta.,4.beta.)]- }\end{array}$ & + & & \\
\hline 204 & $\mathrm{C}_{15} \mathrm{H}_{24}$ & 25.34 & (-)-Aristolene & + & & \\
\hline 226 & $\mathrm{C}_{14} \mathrm{H}_{26} \mathrm{O}_{2}$ & 25.385 & 2,4,7,9-Tetramethyl-5-decyne-4,7-diol & & & + \\
\hline 204 & $\mathrm{C}_{15} \mathrm{H}_{24}$ & 25.636 & Caryophyllene & + & & \\
\hline 202 & $\mathrm{C}_{15} \mathrm{H}_{22}$ & 25.887 & $\begin{array}{c}\text { (3R,4aS,8aS)-8a-Methyl-5-methylene-3-(prop-1-en-2-yl)-1,2,3,4,4a,5,6,8a- } \\
\text { octahydronaphthalene }\end{array}$ & + & & \\
\hline 204 & $\mathrm{C}_{15} \mathrm{H}_{24}$ & 26.114 & Zingiberene & + & & \\
\hline 204 & $\mathrm{C}_{15} \mathrm{H}_{24}$ & 26.228 & .alpha.-Guaiene & + & & \\
\hline 220 & $\mathrm{C}_{15} \mathrm{H}_{24} \mathrm{O}$ & 26.75 & $\begin{array}{c}(1 \mathrm{R}, 2 \mathrm{R}, 4 \mathrm{~S}, 6 \mathrm{~S}, 7 \mathrm{~S}, 8 \mathrm{~S})-8 \text {-Isopropyl-1-methyl-3-methylenetricyclo[4.4.0.02,7] } \\
\text { decan-4-ol }\end{array}$ & + & & \\
\hline 166 & $\mathrm{C}_{11} \mathrm{H}_{18} \mathrm{O}$ & 26.78 & Homomyrtenol & & & + \\
\hline 204 & $\mathrm{C}_{15} \mathrm{H}_{24}$ & 27.021 & Aromandendrene & + & & \\
\hline 204 & $\mathrm{C}_{15} \mathrm{H}_{24}$ & 27.45 & Guaia-6,9-diene & + & & \\
\hline 166 & $\mathrm{C}_{10} \mathrm{H}_{14} \mathrm{O}_{2}$ & 27.572 & Cyclopentaneacetaldehyde, 2-formyl-3-methyl-.alpha.-methylene- & + & & + \\
\hline 202 & $\mathrm{C}_{15} \mathrm{H}_{22}$ & 27.75 & Curcumene & + & & + \\
\hline 154 & $\mathrm{C}_{10} \mathrm{H}_{18} \mathrm{O}$ & 28.013 & Isogeraniol & + & + & + \\
\hline 220 & $\mathrm{C}_{15} \mathrm{H}_{24} \mathrm{O}$ & 28.174 & (2S,3S,6S)-6-Isopropyl-3-methyl-2-(prop-1-en-2-yl)-3-vinylcyclohexanone & + & & \\
\hline 204 & $\mathrm{C}_{15} \mathrm{H}_{24}$ & 28.508 & 4,5-di-epi-aristolochene & + & & \\
\hline 202 & $\mathrm{C}_{15} \mathrm{H}_{22}$ & 28.689 & $\begin{array}{l}\text { (3R,4aS,8aS)-8a-Methyl-5-methylene-3-(prop-1-en-2-yl)-1,2,3,4,4a,5,6,8a- } \\
\text { octahydronaphthalene }\end{array}$ & + & & \\
\hline 206 & $\mathrm{C}_{14} \mathrm{H}_{22} \mathrm{O}$ & 28.858 & 3,5-Di-tert-butylphenol & + & + & + \\
\hline 166 & $\mathrm{C}_{10} \mathrm{H}_{14} \mathrm{O}_{2}$ & 29.455 & 2-(1-Formylvinyl)-5-methylcyclopentanecarbaldehyde & + & + & \\
\hline 220 & $\mathrm{C}_{15} \mathrm{H}_{24} \mathrm{O}$ & 29.465 & Caryophyllene oxide & + & + & + \\
\hline 334 & $\mathrm{C}_{22} \mathrm{H}_{38} \mathrm{O}_{2}$ & 30.136 & Undec-10-ynoic acid, undec-2-en-1-yl ester & + & + & + \\
\hline 222 & $\mathrm{C}_{15} \mathrm{H}_{26} \mathrm{O}$ & 30.361 & Cubenol & + & + & \\
\hline 264 & $\mathrm{C}_{17} \mathrm{H}_{28} \mathrm{O}_{2}$ & 30.811 & Nerolidyl acetate & + & & \\
\hline 222 & $\mathrm{C}_{15} \mathrm{H}_{26} \mathrm{O}$ & 30.984 & Ledol & + & & \\
\hline 302 & $\mathrm{C}_{20} \mathrm{H}_{30} \mathrm{O}_{2}$ & 31.235 & cis-5,8,11,14,17-Eicosapentaenoic acid & & + & + \\
\hline 220 & $\mathrm{C}_{15} \mathrm{H}_{24} \mathrm{O}$ & 31.255 & $\begin{array}{l}\text { 1H-Cycloprop[e]azulen-7-ol, decahydro-1,1,7-trimethyl-4-methylene-, } \\
\text { [1ar-(1a.alpha.,4a.alpha.,7.beta.,7a.beta.,7b.alpha.)]- }\end{array}$ & + & & \\
\hline 328 & $\mathrm{C}_{22} \mathrm{H}_{32} \mathrm{O}_{2}$ & 31.405 & cis-4,7,10,13,16,19-Docosahexanoic acid & & + & + \\
\hline 220 & $\mathrm{C}_{15} \mathrm{H}_{24} \mathrm{O}$ & 31.425 & $\begin{array}{l}\text { 2-((2R,4aR,8aS)-4a-Methyl-8-methylenedecahydronaphthalen-2-yl)prop-2- } \\
\text { en-1-ol }\end{array}$ & + & & \\
\hline 208 & $\mathrm{C}_{15} \mathrm{H}_{28}$ & 31.657 & Selinan & + & & + \\
\hline 222 & $\mathrm{C}_{15} \mathrm{H}_{26} \mathrm{O}$ & 31.79 & Guaiol & + & & \\
\hline 360 & $\mathrm{C}_{24} \mathrm{H}_{40} \mathrm{O}_{2}$ & 31.905 & Undec-10-ynoic acid, tridec-2-yn-1-yl ester & + & + & \\
\hline 252 & $\mathrm{C}_{17} \mathrm{H}_{32} \mathrm{O}$ & 32.313 & 14-Methyl-8-hexadecyn-1-ol & + & + & + \\
\hline
\end{tabular}

Continued... 
Table 3: Cont'd.

\begin{tabular}{|c|c|c|c|c|c|c|}
\hline Molecular Mass & Molecular Formula & R.Time & Name & M & w & $\mathbf{E}$ \\
\hline 204 & $\mathrm{C}_{15} \mathrm{H}_{24}$ & 32.394 & Guaia-6,9-diene & + & + & + \\
\hline 220 & $\mathrm{C}_{15} \mathrm{H}_{24} \mathrm{O}$ & 32.569 & Dehydroxy-isocalamendiol & + & + & \\
\hline 222 & $\mathrm{C}_{15} \mathrm{H}_{26} \mathrm{O}$ & 32.595 & Guai-1(10)-en-11-ol & & & + \\
\hline 220 & $\mathrm{C}_{15} \mathrm{H}_{24} \mathrm{O}$ & 32.73 & (-)-Spathulenol & + & + & + \\
\hline 222 & $\mathrm{C}_{15} \mathrm{H}_{26} \mathrm{O}$ & 32.91 & 1-Naphthalenol, 1,2,3,4,4a,7,8,8a-octahy & + & & \\
\hline 222 & $\mathrm{C}_{15} \mathrm{H}_{26} \mathrm{O}$ & 33.007 & $\begin{array}{l}\text { 2-Naphthalenemethanol, decahydro-.alpha.,.alpha.,4a-trimethyl-8- } \\
\text { methylene-, [2R-(2.alpha.,4a.alpha.,8a.beta.)]- }\end{array}$ & + & & \\
\hline 240 & $\mathrm{C}_{15} \mathrm{H}_{28} \mathrm{O}_{2}$ & 33.075 & Cryptomeridiol & + & & \\
\hline 152 & $\mathrm{C}_{10} \mathrm{H}_{16} \mathrm{O}$ & 33.243 & 2,4,6-Trimethyl-3-cyclohexene-1-carboxaldehyde & + & + & \\
\hline 204 & $\mathrm{C}_{15} \mathrm{H}_{24}$ & 33.355 & $\begin{array}{c}\text { 1H-3a,7-Methanoazulene, 2,3,6,7,8,8a-hexahydro-1,4,9,9-tetramethyl-, } \\
\text { (1.alpha.,3a.alpha.,7.alpha.,8a.beta.)- }\end{array}$ & + & & \\
\hline 168 & $\mathrm{C}_{11} \mathrm{H}_{20} \mathrm{O}$ & 33.867 & (2,2,6-Trimethyl-bicyclo[4.1.0]hept-1-yl)-methanol & + & & + \\
\hline 220 & $\mathrm{C}_{15} \mathrm{H}_{24} \mathrm{O}$ & 34.293 & Caryophyllene oxide & + & + & + \\
\hline 204 & $\mathrm{C}_{15} \mathrm{H}_{24}$ & 34.44 & Longicyclene & + & & + \\
\hline 222 & $\mathrm{C}_{15} \mathrm{H}_{26} \mathrm{O}$ & 34.598 & $\begin{array}{l}\text { 1H-Cycloprop[e]azulen-4-ol, decahydro-1,1,4,7-tetramethyl-, [1ar-(1a. } \\
\text { alpha.,4.alpha.,4a.beta.,7.alpha.,7a.beta.,7b.alpha.)]- }\end{array}$ & + & & + \\
\hline 238 & $\mathrm{C}_{15} \mathrm{H}_{26} \mathrm{O}_{2}$ & 34.75 & Isocalamenediol & & + & + \\
\hline 220 & $\mathrm{C}_{15} \mathrm{H}_{24} \mathrm{O}$ & 34.857 & Longifolenealdehyde & + & & \\
\hline 218 & $\mathrm{C}_{15} \mathrm{H}_{22} \mathrm{O}$ & 34.925 & ,6,6-Trimethyl-2-(3-methylbuta-1,3-dienyl)-3-oxatricyclo[5.1.0.0(2,4)]octane & & & + \\
\hline 290 & $\mathrm{C}_{20} \mathrm{H}_{34} \mathrm{O}$ & 34.989 & Copalol & + & + & \\
\hline 222 & $\mathrm{C}_{15} \mathrm{H}_{26} \mathrm{O}$ & 35.145 & Drim-7-en-11-ol & + & & + \\
\hline 124 & $\mathrm{C}_{9} \mathrm{H}_{16}$ & 35.274 & (2E,4E)-6,6-Dimethyl-2,4-heptadiene & + & & \\
\hline 152 & $\mathrm{C}_{10} \mathrm{H}_{16} \mathrm{O}$ & 35.724 & trans-Verbenol & + & & \\
\hline 222 & $\mathrm{C}_{15} \mathrm{H}_{26} \mathrm{O}$ & 35.849 & Drimenol & + & & + \\
\hline 270 & $\mathrm{C}_{17} \mathrm{H}_{34} \mathrm{O}_{2}$ & 35.92 & Isopropyl myristate & & & \\
\hline 180 & $\mathrm{C}_{12} \mathrm{H}_{20} \mathrm{O}$ & 35.961 & 2,2,6,8-Tetramethyl-7-oxatricyclo[6.1.0.0(1,6)]nonane & + & + & \\
\hline 206 & $\mathrm{C}_{15} \mathrm{H}_{26}$ & 36.268 & $\begin{array}{l}\text { Tricyclo[4.3.0.0(7,9)]nonane, 2,2,5,5,8,8-hexamethyl-, (1.alpha.,6.beta.,7. } \\
\text { alpha.,9.alpha.)- }\end{array}$ & + & & \\
\hline 278 & $\mathrm{C}_{16} \mathrm{H}_{22} \mathrm{O}_{4}$ & 36.47 & Diisobutyl phthalate & & & + \\
\hline 206 & $\mathrm{C}_{14} \mathrm{H}_{22} \mathrm{O}$ & 36.636 & Norpatchoulenol & + & & + \\
\hline 270 & $\mathrm{C}_{17} \mathrm{H}_{34} \mathrm{O}_{2}$ & 37.19 & Methyl 14-methylpentadecanoate & + & & \\
\hline 334 & $\mathrm{C}_{20} \mathrm{H}_{30} \mathrm{O}_{4}$ & 37.62 & Butyl octyl phthalate & + & & + \\
\hline 152 & $\mathrm{C}_{10} \mathrm{H}_{16} \mathrm{O}$ & 38.18 & cis-Chrysanthenol & + & & + \\
\hline 206 & $\mathrm{C}_{15} \mathrm{H}_{26}$ & 38.396 & Patchoulane & + & + & \\
\hline 280 & $\mathrm{C}_{18} \mathrm{H}_{32} \mathrm{O}_{2}$ & 39.042 & cis,cis-Linoleic acid & + & & \\
\hline 296 & $\mathrm{C}_{19} \mathrm{H}_{36} \mathrm{O}_{2}$ & 39.101 & Methyl elaidate & + & & \\
\hline 234 & $\mathrm{C}_{15} \mathrm{H}_{22} \mathrm{O}_{2}$ & 39.265 & Drinenin & + & + & \\
\hline 284 & $\mathrm{C}_{18} \mathrm{H}_{36} \mathrm{O}_{2}$ & 39.349 & Methyl isoheptadecanoate & + & & \\
\hline
\end{tabular}

+ indicates the presence of the listed compound in the indicated extract.

To further quantify the effect of toxin concentration on the induction of mortality, the extracts were serially diluted in artificial seawater to test across a range of concentrations in the Artemia nauplii bioassay. Table 2 shows the $\mathrm{LC}_{50}$ values of the extracts towards $A$. franciscana. $\mathrm{LC}_{50}$ values $>1000 \mu \mathrm{g} / \mathrm{mL}$ towards Artemia nauplii have been defined as being nontoxic. ${ }^{21}$ Based on these results, all extracts tested were deemed nontoxic. Optimised GC-MS parameters were developed and used to examine the T. lanceolata berry extracts. The resultant gas chromatograms are presented in Figure 3. Numerous overlapping peaks were evident in the aqueous berry extract chromatogram (Figure 3a). A total of 79 peaks were detected in the aqueous T. lanceolata berry extract, with the peak eluting at $20.9 \mathrm{~min}$ being the most prominent. Comparison with a phytochemical library putatively identified this peak as the sesquiterpenoid polygodial (Table 3). Numerous overlapping peaks were also evident throughout the chromatogram, with a broad range of retention times between 10-40 $\mathrm{min}$. The presence of peaks throughout the chromatogram 
attest to the wide range of compounds of widely varying polarity extracted with water.

The methanolic berry extract GC-MS chromatogram (Figure 3b) was more complex than the aqueous extract chromatogram. Indeed, a total of 129 peaks were detected in this chromatogram, with major peaks at approximately $14.1,15.1,16.4,19.1,21.0,31.3,31.8$ and $35.0 \mathrm{~min}$. As for the aqueous extract, polygodial (eluting at approximately $21 \mathrm{~min}$ ) was a major component. In addition, a further major peak was evident in the methanolic extract at approximately $16.5 \mathrm{~min}$. A comparison the phytochemical database putatively identified this peak as linalool (Table 3). This compound was also present in the aqueous extract, albeit at a much lower level. Numerous overlapping peaks were also evident throughout the chromatogram, many at retention times corresponding to peaks in the aqueous extract. This indicates that methanol and water extracted many similar components, although many of the lower polarity compounds appear to be more effectively extracted into methanol than water Fewer peaks were evident in the ethyl acetate extract chromatogram (Figure $3 c$ ). Indeed, only 61 unique mass signals were detected in the ethyl acetate extract. As for the other extracts, polygodial was detected in the ethyl acetate berry extract, albeit in substantially lower levels. The ethyl acetate chromatogram also had a major peak present at $19.7 \mathrm{~min}$, which was putatively identified as 2-methyl-2-phenyl-oxirane. Whilst this compound was also detected in both the aqueous and methanolic extracts it was only present in relative abundance in the ethyl acetate extract. Multiple other peaks were also noted in the ethyl acetate extract, many corresponding to peaks in the aqueous and methanolic extracts. However, several peaks in this extract were at different retention times than seen for the more polar methanolic and aqueous extracts.

In total, 156 unique mass signals were noted for the T. lanceolata berry extracts by GC-MS headspace analysis (Table 3). Empirical formulas and putative identifications were achieved for these compounds by comparison against a GC-MS mass and spectral database. A noteable feature of the GC-MS analyses is the diversity of terpenoids compounds noted in the T. lanceolata extracts. The monoterpenoids linalool oxide (16 min), linalool (16.4 min), cymene (16.9 min), $\alpha$-terpineol (19.1 $\mathrm{min}$ ) and isogeraniol $(28 \mathrm{~min})$ as well as the sesquiterpenoids polygodial (21 $\mathrm{min})$, caryophyllene oxide (29.5 and $34.3 \mathrm{~min}$ ) guania-6,9-diene (32.4 $\mathrm{min})$, spathulenol (32.7 $\mathrm{min}$ ) were present in all extracts. However, multiple other terpenoids were present in some but not all extracts. The sesquiterpenoid polygodial was present in all extracts (at approximately $21 \mathrm{~min}$ ) as either the major peak, or one of the largest peaks. A further two sesquiterpenoids were also putatively identified as isomers of caryophyllene oxide. The GC-MS headspace analysis was unable to distinguish which of these isomers corresponded to each peak. One isomer was assumed to be caryophyllene oxide whilst the other compound was assumed to be the structural isomer humulene. Several aliphatic ketocompounds were also detected. Of these, 4-methyl-2-heptanone (11.2 $\mathrm{min})$, 1,1,3,3-tetramethylcyclopentane (11.7 $\mathrm{min}), 6$-methyl-5-hepten-2-one (12.7 $\mathrm{min})$ and 2,2,6,6-tetramethyl-3,5-heptanedione (17.6 min) were present in all extracts. Similarly, the aliphatic compounds dodecane (13.2 $\mathrm{min})$, 4-methylunadecane (16.5 $\mathrm{min})$, nonanoic acid (21.2 $\mathrm{min})$, butyl butoxyacetate (21.8 $\mathrm{min})$, 2,6,11-trimethyldodecane (23 $\mathrm{min}$ ), (6Z)-3,7-dimethyl-6,11-dodecadien-1-ol (24.7 min), undec-10-ynoic acid-undec-2-en-1-yl ester (30.1 $\mathrm{min})$ and the aliphatic ester propanoic acid, 2-methyl-, 3-hydroxy-2,2,4-trimethylpentyl ester (24.2 min) were also present in all T. lanceolata berry extracts. Similarly, the benzene derivatives cumene (13.9 $\mathrm{min}$ ), 1,2-dimethyl-4-ethylbenzene (15.8 $\mathrm{min}$ ), 2-methyl-2-phenyl-oxirane $(20 \mathrm{~min})$ and 3,5-di-tert-butylphenol (28.9 $\mathrm{min})$, were also present in all the berry extracts.

\section{DISCUSSION}

Whilst all the T. lanceolata extracts screened displayed potent $C$. perfringens growth inhibitory activity, the berry extracts generally had greater efficacy than the corresponding leaf extracts. Therefore, the berry extracts were further analysed to determine their phytochemical composition. An examination of the GC-MS headspace metabolomics profile analysis of the aqueous, methanolic, and ethyl acetate berry extracts highlights several interesting compounds. An obvious feature was the number and diversity of terpenoids present in all extracts. The monoterpoid a-terpineol, as well as the sequiterterpenoids polygodial and caryophyllene oxide, was prevalent in all T. lanceolata berry extracts. Indeed, polygodial was the major compound detected in the methanolic and aqueous extracts (based on peak area). This agrees with previous studies which frequently cite polygodial as a major component in T. lanceolata berries (6). Indeed, some studies have reported that polygodial may account for nearly $40 \%$ of commercial $T$. lanceolata essential oil components. ${ }^{22}$

The bacterial growth inhibitory activity of polygodial has been reported in several studies. Polygodial isolated from Warburgia salutaris was reported to be a potent inhibitor of the growth of a panel of bacteria. ${ }^{23}$ Indeed, MIC's of $12.5 \mu \mathrm{g} / \mathrm{mL}$ were reported for polygodial against Staphylococcus aureus and Bacillus subtilis in that study. Whilst less potent, the same study also reported good growth inhibition for polygodial against Staphylococcus epidermidis, Micrococcus luteus, Escherichia coli and Klebsiella pneumoniae, with MIC values generally $\leq 100 \mu \mathrm{g} / \mathrm{mL}$. Other studies have reported little or no antibacterial activity for polygodial against limited panels of bacteria, although many of these studies only tested at relatively low concentrations $(100 \mu \mathrm{g} / \mathrm{mL})) .{ }^{[24]}$ In contrast, more recent studies have demonstrated good bactericidal activity against both Gram-positive and Gram-negative bacteria. ${ }^{25}$ Antifungal efficacy and mechanistic studies of polygodial have been more definitive, with several publications highlighting its potent fungicidal activity. ${ }^{24,26,27}$ Polygoidal appears to exert its antifungal activity by several mechanisms. It nonspecifically disrupts/denatures fungal integral membrane proteins by functioning as a non-ionic surfactant. ${ }^{25}$ It also readily reacts with amino acids (especially cysteine and aromatic amino acids), resulting in further denaturation. As an additional antifungal mechanism, polygoidal may permeate cells by diffusing across the cell membrane. Once inside the cell, polygoidal interacts with various intracellular components and affects metabolic processes. It is possible that polygodial also interacts with bacterial cells in a similar way.

The monoterpene $\alpha$-terpineol was also a common component across all $T$. lanceolata extracts. It is generally believed that monoterpenoids have the most potent broad spectrum bacterial inhibitory activity of all terpenoids compounds, and that this activity is closely linked to their lipophilic character. ${ }^{28} \mathrm{~A}$ variety of monoterpenoids including $\alpha$-terpineol have been shown to have potent antibacterial activity against a panel of Gram-positive and Gram-negative bacteria. ${ }^{28,29}$ The small, hydrophobic nature of monoterpenoids allow them to insert into cytosolic membranes, altering the fluidity and permeability of the membrane, thereby changing the conformation and function of membrane proteins. This consequently interrupts crucial cellular processes including the respiratory chain. Furthermore, the cytoplasmic membrane comprises a cellular barrier to protons and larger ions. ${ }^{30}$ Interestingly, bacteria respond to monoterpenoid exposure by modulating membrane fluidity. ${ }^{31}$ Despite this, the specific antimicrobial mechanisms of monoterpenoids are not yet fully understood.

Interestingly, the non-specific growth inhibitory mechanism of monoterpenoids is inherently difficult for bacteria to counteract/develop resistance to. Indeed, to the best of our knowledge, no bacteria have yet 
developed resistance to any monoterpenoid. Furthermore, as monoterpenoids are generally used in mixtures (extracts, essential oils), it is likely that several growth inhibitory compounds, perhaps with multiple growth inhibitory mechanisms, are used concurrently. This not only enhances the antibacterial efficacy, but also greatly decreases the likelihood of bacteria developing resistance.

A number of other terpenoids were also detected in the T. lanceolata berry extracts by GC-MS headspace analysis. Monoterpenoids were particularly prevalent, with 2,2,6-trimethyl-6-vinyltetrahydropyran (geraniol, Figure 4a), cineole (Figure 4b), a-pinene (Figure 4c), $\beta$-ocimene (Figure $4 \mathrm{~d}$ ), cymene (Figure $4 \mathrm{e}$ ), linalool oxide (Figure $4 \mathrm{f}$ ), linalool (Figure 4g), dihydro- $\gamma$-terpineol (Figure 4h), 4(10)-thujen-3-ol (sabinol) (Figure 4i), trans-linalool-3,7-oxide (Figure 4j), myrtenol (Figure 4k), p-eugenol (Figure 4l), homomyrtenol (Figure 4m), aromandendrene (Figure 4n) and isogeraniol (Figure 4o) detected in the T. lanceolata berry extracts. The bacterial growth inhibitory properties of many of these monoterpenoids have been extensively documented. One study reported noteworthy antibacterial activity for multiple monoterpenoids including $\alpha$-terpineol, geraniol, cineole, $\alpha$-pinene, linalool, sabinene and eugenol against an extended panel of bacteria. ${ }^{32}$ Both Gram-positive and Gram-negative bacteria were susceptible, although the susceptibility varied widely between bacterial species. Unfortunately, that study did not report MIC values, making it difficult to compare efficacies with other compounds in other studies. However, the study was particularly interesting as the authors correlated the inhibitory activity of the monoterpenoids with their structures:

- Generally, terpenoids with a phenolic structure are more highly active than non-phenolic terpenoids.

- The presence of a hydroxyl group further enhances the potency of the terpenoids.

- The position of the hydroxyl group also influences the growth inhibitory potency of the terpenoids.

- Alkyl substitutions reduce the surface tension, altering polarity and subsequently altering bacterial selectivity.

- The addition of an acetate moiety further enhances antibacterial efficacy.

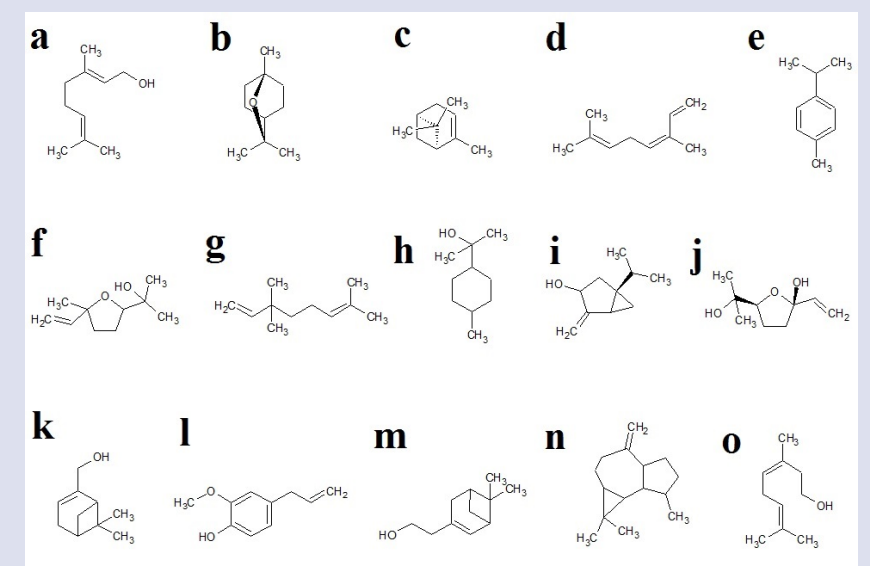

Figure 4: Monoterpenoid components which were detected in the T. lanceolata berry extracts by GC-MS headspace analysis: (a) geraniol, (b), cineole (c), a-pinene, (d) $\beta$-ocimene, (e) p-cymene, (f) linalool oxide, (g) linalool, (h) dihydro- $\gamma$-terpineol, (i) sabinol, (j) trans-linalool-3,7-oxide, (k) myrtenol, (I) p-eugenol, ( $\mathrm{m}$ ) homomyrtenol, ( $\mathrm{n}$ ) aromandendrene and (o) isogeraniol.
- When aldehyde or alcohol moieties are present, the bactericidal activity is increased substantially, possibly via protein degradation, solvating or dehydrating effects.

Similarly, other studies have also reported good antibacterial activity for other monoterpenoids. Eugenol causes bacterial cell wall degradation. ${ }^{33}$ Cymene induces swelling of the cytoplasmic membrane, resulting in bacterial death. ${ }^{34}$ Carvone dissipates the transmembrane $\mathrm{pH}$ gradient and cell potential, thus disrupting general metabolic function. ${ }^{35}$ Cinnamaldehyde is a potent inhibitor of E. coli and Salmonella typhimurium growth. ${ }^{36}$ It is believed that the cinnamaldehyde carbonyl group has affinity for bacterial decarboxylases, preventing their function. ${ }^{37}$

Several sesquiterpenoids including $\alpha$-cubenene (Figure $5 a$ ), caryophyllene (Figure 5b), zingiberene (Figure 5c), a-guaiene (Figure 5d), cubenol (Figure 5e), ledol (Figure 5f), spathulenol (Figure 5g), guaiol (Figure 5h), guai-1(10)-en-11-ol (Figure 5i), longicyclene (Figure 5j), isocalamenediol (Figure 5k), longifolenealdehyde (Figure 5l) were also present in the T. lanceolata berry extracts. The potent antibacterial activity of sesquiterpenoid lactones isolated from red algae (Laurencia spp.) has been reported, with MIC values $10-40 \mu \mathrm{g} / \mathrm{mL}$ against Chromobacterium violaceum, Erwinia spp., two Proteus species. and two Vibrio species. ${ }^{[38]}$ Whilst less potent, good growth inhibitory activity has also been reported for selina-4,11(13)-dien-3-on-12-oic acid isolated from Varthemia iphionoides against Bacillus cereus, B. subtilis, E. coli, Micrococcus luteus, Salmonella enteritides and $S$. aureus. ${ }^{39}$ However, the sesquiterpenoids are generally considered to be less potent bacterial growth inhibitors than the monoterpenoids. ${ }^{38}$ Instead, the sesquiterpenoids appear to exert their antibacterial activities through interactions with other phytochemicals. The sesquiterpenoids nerolidol, farnesol, bisabolol and apritone have been reported to enhance the susceptibility of bacteria to other antibiotic compounds, possibly by increasing the permeability of the bacterial membrane to those compounds. ${ }^{40}$ Thus, as well as having moderate growth inhibitory activity themselves, it is likely that the T. lanceolata sesquiterpenoids enhance the activity of the other antibacterial compounds in the extracts. Whilst reports of antibacterial activity are lacking for many of the other sesquiterpenoids, some have been identified in bacterial growth inhibitory plant extracts. ${ }^{41-45}$ a

e

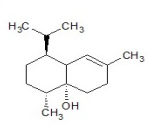

i

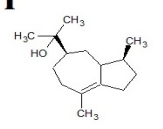

b

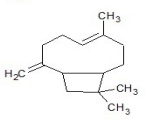

$\mathbf{f}$

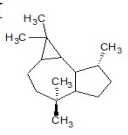

$\mathbf{j}$

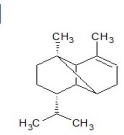

c<smiles>CC(C)=CCCC(C)c1ccc(C)cc1</smiles>

$\mathbf{g}$

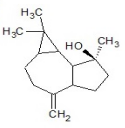

$\mathbf{k}$

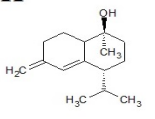

d $\underbrace{\mathrm{CH}_{3}}_{\mathrm{CH}_{3}}$

h<smiles>CC1CCC2(C)CCC(C(C)C2)C1C</smiles>

$\mathbf{I}$

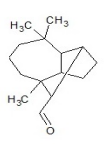

Figure 5: Sesquiterpenoid components detected in the T. lanceolata berry extracts by GC-MS headspace analysis: (a) a-cubenene, (b) caryophyllene, (c) zingiberene, (d) a-guaiene, (e) cubenol, (f) ledol, (g) spathulenol, (h) guaiol, (i) guai-1(10)-en-11-ol, (j) longicyclene, (k) isocalamenediol and (I) longifolenealdehyde. 
Our study demonstrates that $T$. lanceolata berry extracts contain a variety of different compounds which may contribute to the $C$. perfringens growth inhibitory activity. Furthermore, a comparison between the metabolomics profiles of the extracts has highlighted several compounds of interest. However, it is unlikely that any single molecule is solely responsible for the T. lanceolata berry $C$. perfringens growth inhibitory activity. Instead, it is more likely that several compounds contribute to this activity. Furthermore, it is possible that synergistic interactions between the various bioactive components may be potentiating the growth inhibitory activity of the individual components, increasing their efficacy. At the very least, the presence of numerous molecules with growth inhibitory activity indicates that these extracts are likely to function by pluripotent pathways. Further studies are warranted to test the activity of the phytochemical compounds, both individually and in combinations.

\section{ACKNOWLEDGEMENT}

We are grateful to Jane Gifkins and John Bates for the gift of the C. perfringens clinical strain used in these studies. Financial support for this work was provided by the Environmental Futures Research Institute and the School of Natural Sciences, Griffith University, Australia.

\section{CONFLICTS OF INTEREST}

The authors declare that they have no conflict of interest.

\section{ABBREVIATION USED}

DMSO: Dimethyl sulfoxide; $\mathbf{L C}_{50}$ : The concentration required to achieve 50 \% mortality; MIC: Minimum inhibitory concentration.

\section{REFERENCES}

1. Stevens DL, Bryant AE. The role of clostridial toxins in the pathogenesis of gas gangrene. Clin Infect Dis. 2002;35(Suppl 1):S93-100. DOI: 10.1086/341928 https://doi.org/10.1086/341928.

2. Tanaka D, Isobe J, Hosorogi S, Kimata K, Shimizu M, Katori K, et al. An outbreak of food-borne gastroenteritis caused by Clostridium perfringens carrying the cpe gene on a plasmid. Jpn J Infect Dis. 2003;56(3):137-9. PMid:12944687.

3. Freedman JC, Theoret JR, Wisniewski JA, Uzal FA, Rood JI, McClane BA. Clostridium perfringens type A-E toxin plasmids. Res Microbiol. 2015;166(4):264-79. 10.1016/j.resmic.2014.09.004; PMid:25283728 PMCid:PMC4383721.

4. Aggelidakis J, Lasithiotakis K, Topalidou A, Kouroumpas J, Kouvidis G, Katonis P. Limb salvage after gas gangrene: a case report and review of the literature. World J Emerg Surg. 2011;6(1):28. https://doi.org/10.1186/1749-7922-6-28; PMid:21846405 PMCid:PMC3182882.

5. Bakker DJ. Clostridial myonecrosis (gas gangrene). Undersea Hyperb Med. 2012;39(3):731-7. PMid:22670554.

6. Cock IE. The phytochemistry and chemotherapeutic potential of Tasmannia lanceolata (Tasmanian pepper): A review. Pharmacog Commn. 2013;3(4):13-25.

7. Cock IE. Medicinal and aromatic plants - Australia. In Ethnopharmacology section, Biological, Physiological and Health Sciences 2011; Encyclopedia of Life Support Systems (EOLSS), Developed under the auspices of UNESCO, EOLSS Publishers, Oxford, UK. (http://www.eolss.net).

8. Netzel M, Netzel G, Tian Q, Schwartz S, Konczak I. Native Australian fruits - a novel source of antioxidants for food. Innov Food Sci Emerg Technol. 2007;8(3):339-46. DOI: 10.1016/j.ifset.2007.03.007.

9. Winnett V, Boyer H, Sirdaarta J, Cock IE. The potential of Tasmannia lanceolata as a natural preservative and medicinal agent: antimicrobial activity and toxicity. Pharmacog Commn. 2014;4(1):42-52. DOI: 10.5530/pc.2014.1.7

10. Cock IE, Winnett V, Sirdaarta J, Matthews B. The potential of selected Australian medicinal plants with anti-Proteus activity for the treatment and prevention of rheumatoid arthritis. Pharmacog Mag. 2015;42(Supp1):S190-208. DOI: 10.4103/0973-1296.157734

11. Lee CJ, Wright MH, Arnold MSJ, Greene AC, Cock IE. Inhibition of Streptococcus pyogenes growth by native Australian plants; New approaches towards the management of impetigo, pharyngitis and rheumatic heart disease. Pharmacog Commn. 2016;6(3):164-73. DOI: 10.5530/pc.2016.3.6

12. Wright $\mathrm{MH}$, Greene AC, Cock IE. Inhibition of Bacillus anthracis growth by Australian native plants used traditionally as antibacterial medicines. Pharmacog $\mathrm{J}$. 2015;7(6):389-96. DOI: 10.5530/pj.2015.6.13

13. Wright MH, Sirdaarta J, Matthews B, Greene AC, Cock IE. Growth inhibitory activity of Kakadu plum extracts against the opportunistic pathogen Clostridium perfringens: New leads in the prevention and treatment of clostridial myonecrosis Pharmacog J. 2016;8(2):144-53. DOI: 10.5530/pc.2016.2.7

14. Sirdaarta J, Matthews B, White A, Cock IE. GC-MS and LC-MS analysis of Kakadu plum fruit extracts displaying inhibitory activity against microbial triggers of multiple sclerosis. Pharmacog Commn. 2015;5(2):100-15. DOI: 10.5530/pc.2015.2.2

15. Cock IE, van Vuuren SF. The potential of selected South African plants with antiKlebsiella activity for the treatment and prevention of ankylosing spondylitis. Inflammopharmacology. 2015;23(1):21-35. DOI: 10.1007/s10787-014-0222-z

16. Kalt FR, Cock IE. Gas chromatography-mass spectroscopy analysis of bioactive Petalostigma extracts: Toxicity, antibacterial and antiviral activities. Pharmacog Mag. 2014;10(Suppl 1):S37-49. DOI: 10.4103/0973-1296.127338

17. Vesoul J, Cock IE. The potential of Bunya Nut as an antibacterial food agent. Pharmacog Commn. 2012;2(1):72-79. DOI: 10.5530/pc.2012.1.13

18. Ruebhart D, Wickramasinghe W, Cock IE. Protective efficacy of the antioxidants vitamin $E$ and Trolox against Microcystis aeruginosa and microcystin-LR in Artemia franciscana nauplii. J Toxicol Environ Health, Part A. 2009:72(24):1567-75. DOI: 10.1080/15287390903232459

19. Wright MH, Lee CJ, Pollock CE, Greene AC, Cock IE. Growth inhibitory activity of Kakadu Plum extracts against the opportunistic pathogen Clostridium perfringens; New leads in the prevention and treatment of clostridial myonecrosis Pharmacog Commn. 2016;6(2):93-99. DOI: 10.5530/pj.2016.2.8

20. Biggs I, Sirdaarta J, White A, Cock IE. GC-MS analysis of frankincense extracts which inhibit the growth of bacterial triggers of selected autoimmune diseases. Pharmacog Commn. 2016;6(1):10-22. DOI: 10.5530/pc.2016.1.3.

21. Cock IE, Ruebhart DR. Comparison of the brine shrimp nauplii bioassay and the ToxScreen-II test for the detection of toxicity associated with Aloe vera (Aloe barbadensis Miller) leaf extract. Pharmacog Res. 2009;1(2):98-101.

22. Menary RC. Mountain pepper extract, Tasmannia lanceolata: quality stabilisation and registration. A report for the Rural Industries Research and Development Corporation (RIRDC). Barton ACT 2003; (http://www.rirdc.gov.au/reports/ EOI/02-148.pdf)

23. Rabe T, Van Staden J. Isolation of an antibacterial sesquiterpenoid from Warburgia salutaris. J Ethnopharmacol. 2000;73(1):171-4. DOI: 10.1016/ S0378$8741(00) 00293-2$

24. Taniguchi M, Adachi T, Akihiko OSK, Shigeo K, Sachihiko I, Isao K. Structureactivity relationship of the Warburgia sesquiterpene dialdehydes. Agric Biol Chem. 1984:48:73-8. DOI: 10.1271/bbb1961.48.73.

25. Kubo I, Fujita K, Lee SH, Ha TJ. Antibacterial activity of polygodial. Phytother Res. 2005;19:1013-7. https://doi.org/10.1002/ptr.1777 ; PMid:16372365

26. Alves TM, Ribeiro FL, Kloos H, Zani CL. Polygodial, the fungitoxic component from the Brazilian medicinal plant Polygonum punctatum. Mem Inst Owwaldo Cruz 2001;96(6):831-3. https://doi.org/10.1590/S0074-02762001000600016.

27. Lee SH, Lee JR, Lunde CS, Kubo I. In vitro antifungal susceptibilities of Candida albicans and other fungal pathogens to polygodial, a drimane sesquiterpene dialdehyde. Planta Med. 1999;65(03):204-8. DOI: 10.1055/s-1999-13981.

28. Paduch R, Kandefer-Szerszen M, Trytek M, Fiedurek J. Terpenes: substances useful in human healthcare. Arch Immunol Ther Exp. 2007;55(5):315-27. DOI: 10.1007/s00005-007-0039-1.

29. Zenegin $H$, Baysal $A H$. Antibacterial and antioxidant activity of essential oil terpenes against pathogenic and spoilage-forming bacteria and cell structureactivity relationships evaluated by SEM microscopy. Molecules. 2014;19:17773-98. DOI: 10.3390/molecules191117773; PMid:25372394.

30. Cristani M, D’Arrigo M, Mandalari G, Castelli F, Sarpietro MG, Micieli D. Interaction of four monoterpenes contained in essential oils with model membranes: implications for their antibacterial activity. J Agric Food Chem. 2007;55(15):6300-8. https://doi.org/10.1021/jf070094x PMid:17602646.

31. Di Pasqua R, Hoskins N, Betts G, Mauriello G. Changes in membrane fatty acid composition of microbial cells induced by addition of thymol, carvacrol, limonene, cinnamaldehyde, and eugenol in the growing media. J Agric Food Chem 2006;54(7):2745-9. DOI: 10.1021/jf052722l; PMid:16569070.

32. Dorman HJD, Deans SG. Antimicrobial agents from plants: antibacterial activity of plant volatile oils. J Appl Microbiol. 2000;88(2):308-16. DOI: 10.1046/j.1365-2672.2000.00969.x

33. Thoroski J, Blank G, Bilideris C. Eugenol induced inhibition of extracellular enzyme production by Bacillus cereus. J Food Prot. 1989:52(6):399-403. DOI: 10.4315/0362-028X-52.6.399.

34. Ultee A, Bennink MHJ, Moezelaar R. The phenolic hydroxyl group of carvacro is essential for action against the food-borne pathogen Bacillus cereus. Appl Environ Microbiol. 2002;68(4):1561-8. DOI: 10.1128/AEM.68.4.1561-1568.2002; PMid:11916669 PMCid:PMC123826.

35. Oosterhaven K, Poolman B, Smid EJ. S-carvone as a natural potato sprout inhibiting, fungistatic and bacteriostatic compound. Ind Crops Prod. 1995;4(1):23-31 DOI: 10.1016/0926-6690(95)00007-Y

36. Helander IM, Alakomi HL, Latva-Kala K, Mattila-Sandholm T, Pol I, Smid EJ, Gorris LGM, von Wright A. Characterization of the action of selected essential components on gram-negative bacteria. J Agric Food Chem. 1998:46(9):3590-5. DOI: $10.1021 / \mathrm{jf980154m}$.

37. Wendakoon CN, Sakaguchi M. Inhibition of amino acid decarboxylase activity in Enterobacter aerogenes by active compounds in spices. J Food Prot. 
1995;58(3):280-3. DOI: 10.4315/0362-028X-58.3.280

38. Vairappan CS, Suzuki M, Ishii T, Tatsufumi O, Tsuyoshi A, Michio M. Antibacterial activity of halogenated sesquiterpenes from Malaysian Laurencia spp. Phytochemistry. 2008;69(13):2490-4. DOI: 10.1016/j.phytochem.2008.06.015; PMid:18718619

39. Al-Dabbas MM, Hashinaga F, Abdelgaleil SA, SuganumaT, Akiyama K, Hayashi H. Antibacterial activity of an eudesmane sesquiterpene isolated from common Varthemia, Varthemia iphionoides. J Ethnopharmacol. 2005;97(2):237-40. DOI: 10.1016/j.jep.2004.11.007; PMid:15707759

40. Brem-Stecher B, Johnson EA. Sensitisation of Staphylococcus aureus and Escherichia coli to antibiotics by the sesquiterpenoids nerolidol, farnesol, bisabolol, and apritone. Antimicrob Agents Chemother. 2003;47(10):3357-60. DOI: 10.1128/AAC.47.10.3357-3360.2003.

41. Goni P, López P, Sánchez C, Gómez-Lusa R, Becerrila R, Nerínb C. Antimicrobial activity in the vapour phase of a combination of cinnamon and clove essential

\section{GRAPHICAL ABSTRACT}

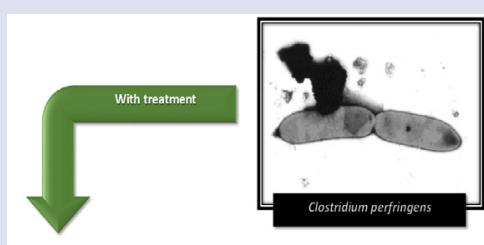

0
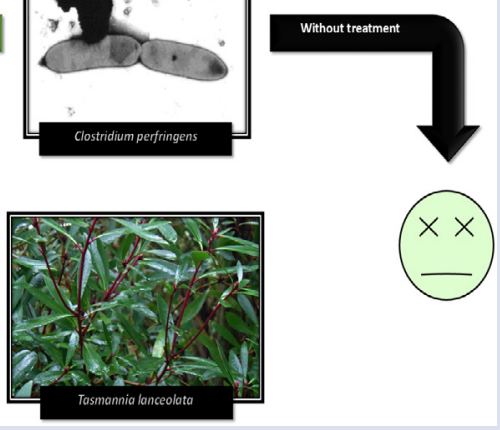

\section{AUTHOR PROFILE}

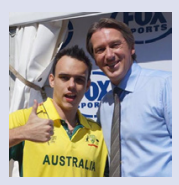

Dr. Mitchell Henry Wright is a postdoctoral researcher at Oregon Health \& Science University in Portland, Oregon (USA) where he works on investigating Mn(III) transformations in aquatic systems. Specifically, his research focuses on manganese oxidation/reduction by bacteria and how these organisms influence the geochemical cycling of the metal. His previous postdoctoral posting involved investigating the potential of Australian native plants in the treatment and prevention ofvarious pathogenic bacteria. This has resulted in several publications between both disciplines.

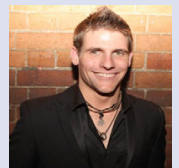

Mr. Cameron Jay Lee completed his Bachelor of Science with Honours in 2016 and is currently beginning his $\mathrm{PhD}$. His research involves the investigation of thermophilic anaerobes that utilize toxic metals in anaerobic respiration (including uranium and arsenic). He has extensive experience in anaerobic cultivation/isolation andin numerous analytical techniques associated with heavy metal analysis.

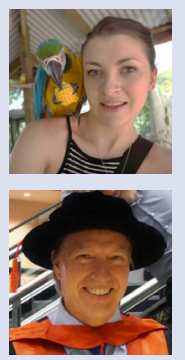

Ms. Megan Sarah Jean Arnold is currently undertaking her PhD in Tropical Parasitology at Griffith University's EskitisInstitute for Drug Discovery with a focus on the identification and development of novel chemoprophylactic agents for malaria. Her other research interests include investigating Australian high antioxidant plants for theirantibacterial capabilities.

Dr. Anthony Carlson Greene is a senior lecturer and researcher at Griffith University, Brisbane Australia. He obtained his PhD in Microbiology from the University of New South Wales and focuses on extreme environments, Bioremediation and Geomicrobiology. His specific interests include the microbial ecology of thermophilic, saline and alkaliphilic environments and the mechanisms and industrial potential of extremophilic bacteria contained therein.

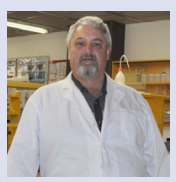

Dr lan Edwin Cock leads a research team in the Environmental Futures Research Institute and the School of Natural Sciences at Griffith University, Australia. His research involves bioactivity and phytochemical studies into a variety of plant species of both Australian and international origin, including Aloe vera, South Asian and South American tropical fruits, as well as Australia plants including Scaevolaspinescens, Pittosporum phylliraeoides, Terminalia ferdinandiana (Kakadu plum), Australian Acacias, Syzygiums, Petalostigmas and Xanthorrhoea johnsonii(grass trees). This range of projects has resulted in nearly 200 scientific publications in a variety of international peer reviewed journals.

Cite this article : Wright MH, Lee CJ, Arnold MSJ, Shalom J, White A, Greene AC, Cock IE. GC-MS analysis of Tasmannia lanceolata extracts which inhibit the growth of the pathogenic bacterium Clostridium perfringens. Pharmacog J. 2017;9(5):626-37. 